\section{Hipatia Press}

www.hipatiapress.com

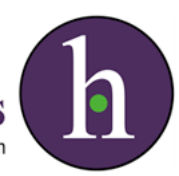

Instructions for authors, subscriptions and further details:

http://rise.hipatiapress.com

\title{
Creación de Recursos Educativos Digitales: Reflexiones sobre Innovación Educativa con TIC
}

Isabel Pérez-Ortega ${ }^{1}$

1) University of Cantabria, Spain

Date of publication: June $25^{\text {th }}, 2017$

Edition period: June 2017-October 2017

To cite this article: Pérez-Ortega, I. (2017). Creación de Recursos Educativos Digitales: Reflexiones sobre Innovación Educativa con TIC. International Journal of Sociology of Education, 6(2), 244-268. doi: 10.17583/rise.2017.2544

To link this article: http://dx.doi.org/10.17583/rise.2017.2544

PLEASE SCROLL DOWN FOR ARTICLE

The terms and conditions of use are related to the Open Journal System and to Creative Commons Attribution License (CC-BY) 


\section{Practicing the Creation of Digital Educational Resources: Reflections about Educational Innovation with ICT}

Isabel Pérez-Ortega

University of Cantabria

(Received: 2 February 2017; Accepted: 22 May 2017; Published: 25 June 2017)

\section{Abstract}

This text analyzes the experience of teaching innovation project called "Development of audiovisual products as digital educational resources" which was developed with graders of Social Communication at the Autonomous Metropolitan University Xochimilco (UAM-X) in Mexico City, Mexico; as well as, graders of Teaching at the University of Cantabria (UC) in Santander, Spain. Whereby, the author (using a social-critical perspective) refers, observes and interprets the phases that formed an exercise that aims the creation and broadcasting of videos that in the future can be transformed into digital educational resources. Then, using the perspective of sociology of education, which attaches great importance to social and cultural factors around educational phenomena, the text concludes that the activities of this project are useful because they promote an academic dialogue about the necessity to change the paradigms into the roles of teachers and students in the process of transmission of digitized knowledge; the requirement to educate them in understanding the language of ICT and finally, to recognize their own abilities to make changes and / or improvements to the school dynamics.

Keywords: higher education, educational innovation, digital educational resources, sociology of education, ict in educational contexts 


\section{Creación de Recursos Educativos Digitales: Reflexiones sobre Innovación Educativa con TIC}

Isabel Pérez-Ortega

University of Cantabria

(Recibido: 2 Febrero 2017; Aceptado: 22 Mayo 2017; Publicado: 25 Junio 2017)

\section{Resumen}

Este texto analiza la experiencia del proyecto de innovación educativa Elaboración de productos audiovisuales como recursos educativos digitales, el cual fue desarrollado con estudiantes de los grados de Comunicación Social de la Universidad Autónoma Metropolitana -Xochimilco (UAM-X) en la Ciudad de México, así como los alumnos de Magisterio en Educación Primaria y de Magisterio en Educación Infantil de la Universidad de Cantabria (UC) en Santander, España. Para ello, se refieren, observan e interpretan desde una perspectiva socio-crítica las fases que integraron un ejercicio teórico-práctico que impulsó la creación y la difusión de videos con vistas a configurarse en recursos educativos digitales. Luego, desde la perspectiva de la Sociología de la Educación, la cual dota de gran importancia a los factores sociales y culturales alrededor de los fenómenos educativos, se concluye que las actividades que integran el Proyecto favorecen la formación de los alumnos porque permiten: a) dialogar (académicamente) sobre la necesidad de modificar los paradigmas en los roles de profesores y estudiantes en el proceso de transmisión del conocimiento digitalizado; b) señalar la necesidad de educar en la comprensión de los lenguajes de las TIC; y finalmente; c) reconocer sus propias capacidades de realizar cambios y/o mejoras a la dinámica escolar.

Palabras clave: educación superior, innovación educativa, recursos educativos digitales, sociología de la educación, tic en contextos educativos 


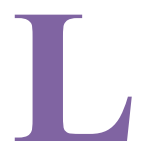

as discusiones sobre el acceso y el uso de las Tecnologías de la Información y la Comunicación (TIC) en contextos escolares son frecuentes. También los debates en torno la potencialidad de estos instrumentos para la participación más activa de docentes y discentes, no solo como usuarios sino como creadores de contenidos educativos. Este texto, mediante la reseña, la observación y la interpretación del proyecto Elaboración de productos audiovisuales como recursos educativos digitales (en adelante Proyecto) pretende ser un aporte al debate académico sobre: a) la utilidad de desarrollar actividades teórico-prácticas con recursos TIC, ya que estas permiten dialogar sobre la importancia de transformar los paradigmas en los roles de profesores y alumnos en el proceso de transmisión del conocimiento digitalizado; b) la necesidad de educar en la comprensión de los lenguajes de las TIC, más que en la adquisición de habilidades técnicas, ya que como se insiste desde las teorías de la reproducción del ámbito sociológico, será fundamental que las instituciones educativas doten a los alumnos de herramientas que les permitan significar de forma crítica los contenidos que ofrecen estos medios de interacción social y educativa en formatos virtuales; y c) el beneficio para que alumnos y profesores constituye la identificación de sus propias capacidades de realizar cambios y/o mejoras a la dinámica escolar. Así, el propósito del texto es, mediante el análisis y la interpretación las actividades de proyecto Elaboración de productos audiovisuales como recursos educativos digitales, señalar el papel de las TIC como herramientas y como canales, pero sobre todo como soportes de discursos simbólicos que (cada vez más) forman parte del proceso de producción, reproducción y distribución de las estructuras, la organización y los saberes institucionalizados; y luego, su importancia en la búsqueda de cambios y mejoras en el proceso de transmisión de conocimientos escolares, a través (en este caso) de la creación de recursos educativos digitalizados como discursos alternativos al dominante.

Siguiendo a Martínez González (2007, p.13) se considera de gran relevancia llevar a cabo investigaciones sobre realidades educativas para (a través de la innovación) intentar cambiar y/o mejorar algunas prácticas educativas. Para esta autora, desarrollar y observar sistemáticamente este tipo de acciones permite "analizar con rigurosidad y objetividad una situación educativa” que servirá para realizar valoraciones y diagnósticos 
sobre la misma. En este caso se observa un proyecto que derivó en la elaboración de productos audiovisuales como recursos educativos mediados por la tecnología digital por parte de alumnos de 83 alumnos de Grado de Educación Infantil y Primaria de la Universidad de Cantabria (UC en Santander, España) y de 60 alumnos de Grado de Comunicación Social de la Universidad Autónoma de México - Xochimilco (UAM-Xochimilco, en Ciudad de México), durante los cursos académicos 2014-15 y 2015-16; y que siguió un método de enseñanza- aprendizaje que pretendió colocar a los alumnos como productores de discursos audiovisuales para contextos educativos. "El protagonismo es una oportunidad para que [los estudiantes] contribuyan a seleccionar recursos disponibles en la red según preferencias y necesidades, contribuyendo asimismo a la elaboración de contenidos y a la creación de conocimiento" (Durall, Gros, Maina, et. al, 2012, p. 17).

Así, la observación del Proyecto como un conjunto de actividades que permitieron experimentar el uso de TIC en contextos educativos como un recurso tecnológico y como una metodología de enseñanza-aprendizaje que posiciona al alumnado como productor de conocimientos, se rigió bajo los preceptos de la línea de investigación socio-crítica que vincula la teoría con la práctica y cuyo objetivo es "formar a personas para que desarrollen su capacidad de reflexión crítica y les permita analizar sus propio contexto y realidad cotidiana” (Martínez González, 2007, p. 33). Así, el Proyecto se desarrolló en el espacio de dos asignaturas de grado con perspectiva sociológica común, pero en contextos geográficos diferenciados.

En la primera parte del texto se establecen los razonamientos teóricos utilizados para observar e interpretar el Proyecto. En la segunda parte se exponen los elementos que integraron el proyecto. La última parte comprende la interpretación y la valoración de los resultados del Proyecto, es decir, de la experiencia de creación de grabaciones audiovisuales como recursos didácticos digitales y sus implicaciones en contextos educativos.

\section{Fundamentos Teóricos: Tic e Innovación Educativa}

El Proyecto es en esencia un ejercicio práctico. El diseño, creación y presentación de los productos digitales fueron experiencias empíricas; sin embargo, esto no excluye que sus fundamentos teóricos encuentren cabida en las reflexiones académicas sobre el uso de TIC en contextos educativos. 


\section{Pérez-Ortega-Recursos Educativos Digitales}

Una de las funciones sociales de la escuela es la de complementar los procesos de socialización de los individuos. En el caso de la educación superior, es fundamental introducir experiencias formativas que contribuyan a facilitar las exigencias de interpretación de los lenguajes de los productos y los canales digitales. Además, propicia el aprendizaje de pensamientos críticos sobre el acceso, uso, consumo y producción de los contenidos de los medios audiovisuales; o incluso respecto a la promoción de la responsabilidad social a través del uso, disfrute y aprovechamiento de estos. Por ello, el Proyecto propone la elaboración de productos audiovisuales como recursos educativos, para ejemplificar que, como discursos alternativos al dominante, podrían mejorar y/o cambiar algunos aspectos de las metodologías docentes de nivel educativo postobligatorio. A continuación, se delimita este concepto y se destaca su importancia como estructura de legitimación de determinadas formas de interacción social.

Como punto de partida, se establece el currículo escolar como un conjunto de materiales (tangibles o digitales) que promueve un particular tipo de aprendizaje. Estos elementos poseen la capacidad de incidir en la producción, la reproducción y la distribución del saber, porque desarrolla una precisa función social en la construcción de la cultura "y no sólo en sentido genérico, sino también desde una perspectiva institucional atenta a las singulares dinámicas que modulan su recreación y creación de la escuela" (Romero y Luis, 2008). Así, desde una la línea de pensamiento del análisis de la cultura y de las representaciones sociales, el currículo escolar es un conjunto de prácticas políticas, sociales y pedagógicas necesarias para la reproducción de específicos conocimientos, habilidades o destrezas, actitudes y valores. Es decir, es una construcción social y política capaz de dotar de significado a ciertos valores y principios que integran, justifican y legitiman una determinada forma de organización de los contenidos escolares. El currículo escolar posee gran importancia en el proceso de producción y reproducción de conocimientos, porque al referir un sistema de valores, normas, convenciones, sobrentendidos, prácticas diarias, rutinas y disposiciones organizativas que existen en un centro escolar concreto, tiene capacidad de influir en la manera de pensar e incluso en la forma de interaccionar de los distintos integrantes de los centros escolares.

En contextos educativos (occidentales) mediados por las TIC, cada vez más se presentan cambios que impactan (desigualmente) las formas de 
organización y las metodologías docentes tanto en los niveles de enseñanza básica como en la postobligatoria. Cada vez más, la llamada cultura formativa-educativa

que coloca la centralidad del aprendizaje en el estudiante y que se fundamenta en el uso de tecnologías" lleva a la necesidad de reconocer "la capacidad y responsabilidad del estudiante para aprender cierta autonomía; una autonomía diferente de la labor del docente y una infraestructura tecnológica que favorezca la participación, el trabajo colaborativo en redes de aprendizaje y las comunidades de práctica (Durall, Gros, Maina, et. al., 2012, p.18).

La integración de recursos tecnológicos y la digitalización de la información en las instituciones de educación superior, exige la reconfiguración de las representaciones colectivas $\mathrm{y}$, por ende, de las estructuras y de las prácticas en cuanto la producción, el procesamiento y la distribución del conocimiento. Es decir, la informatización de los entornos educativos desvela como trasfondo un necesario cambio cultural en las orientaciones de las acciones y de los recursos pedagógicos. Es necesario modificar las estructuras de las instituciones superiores, ya que "muchas de las dificultades para incorporar las TIC radican en los modelos organizativos actuales de las organizaciones educativas. Las universidades continúan implementando modelos educativos tradicionales que dificultan la creación de nuevos escenarios de comunicación y educación con las TIC" (Durall, Gros, Maina, et. al., 2012, p. 3). Así, es fundamental desarrollar acciones de alfabetización mediática que pretendan, no solo promover el acceso a los medios de comunicación, sino que sean capaces de trascender el concepto de alfabetización verbal y de lectoescritura, para incluir lenguajes y formas de expresión basadas en la imagen digital fija y en movimiento. Siguiendo a Gutiérrez y Tyner (2012, p. 10), “en este proceso de transformación, la alfabetización mediática contempla la aparición de nuevos medios de comunicación dentro de los contextos históricos, culturales, sociales y económicos de la alfabetización".

Al observar el proceso de enseñanza-aprendizaje desde algunos de los fundamentos de la sociología de la educación (que consideran relevantes los entornos económicos y políticos - pero sobre todo los sociales y culturalesalrededor de las acciones educativas, así como a las relaciones de fuerza que 
alrededor de las mismas) se destaca la importancia que tienen otras agencias de socialización, además de la escuela. De esta manera, siguiendo a Pérez y Delgado (2012) se considera que la alfabetización digital (nombrada así de manera genérica) en contextos de educación básica (pero también posobligatoria) ha de impulsar el desarrollo de habilidades no solo de acceso, sino de uso e interpretación de herramientas TIC, de manera que permitan al alumnado interpretar y reelaborar la información a su disposición.

Las instituciones de educación superior deben tener en cuenta el valor único que cada persona agrega a un mundo en el que la información está en todos partes. En este nuevo escenario la capacidad de evaluar la credibilidad de la información y la creación de sentido son primordiales (Durall, Gros, Maina, et. al., 2012, p. 17).

Para ello es necesario contar con contextos extraescolares que permitan la utilización de estos conocimientos y habilidades, ya que de otra forma la alfabetización digital no resulta en estrategia de inclusión socio-tecnológica. Para que así fuera, tendrían que coincidir factores económicos, políticos, sociales, culturales, ya que "la situación política y sociocultural de un grupo social forma sus normas y valores, lo cual a su vez influye en el significado que se le da a un artefacto" (Pinch y Bijker, 1987 en Perazzo, 2008). Es decir, será fundamental dotar de relevancia, además de las políticas públicas que podrían favorecer o no la dotación de recursos tecnológicos, a los actores, quienes mediante sus prácticas en contextos educativos, son quienes elaboran y reelaboran los conceptos alrededor de las TIC.

Dado que el Proyecto pretendió elaborar videos originales para contextos educativos formales de nivel superior, a continuación se presenta una concisa descripción de la noción recurso didáctico digital como parte del currículo escolar: se entiende como el conjunto de materiales digitalizados, producidos con el objetivo de facilitar el desarrollo de actividades de aprendizaje. Así, su objetivo es favorecer la transmisión de conocimientos, la adquisición de habilidades e incluso el fomento de determinados valores (Zapata, 2012). Se diferencian de otros recursos educativos (como por ejemplo los documentos tangibles -libros u otros materiales impresos-) porque constituyen discursos multimedia, es decir, están nutridos, además de textos o imágenes, de sonido, videos, simuladores, laboratorios virtuales, 
bases de datos y gráficos interactivos. Utilizan un formato que ofrece una red de conexiones entre los bloques de información, por lo cuales se puede "navegar", siguiendo itinerarios personalizados y su lectura exige dispositivos electrónicos y disponer de conexión a Internet. (Zapata, 2012).

En la actualidad estos instrumentos resultan de gran utilidad para la adquisición de conocimientos. Para Zapata (2012), entre las ventajas de la utilización de los recursos, destaca su formato en cuanto que: permite la presentación de los contenidos (animaciones y tutoriales audiovisuales) de manera más motivadora; admite el uso de simuladores y laboratorios virtuales, que representan situaciones reales o ficticias a las que no es posible tener acceso en el mundo real cercano; permite modular el ritmo de aprendizaje en cuanto que cada estudiante puede consultar los materiales de forma personal; algunos ofrecen la posibilidad de acceso abierto

donde los autores tienen la potestad de conceder una forma de licencia CreativeCommons a sus recursos educativos que publican en la WEB, o de compartirlos con otros usuarios en espacios de la WEB 2.0 y en espacios orientados a generar redes sociales (Zapata, 2012, p. 4).

El uso y la creación de TIC en contextos educativos formales implican la creación, la búsqueda y la selección de recursos educativos digitales en función de objetivos particulares. Es decir, los recursos educativos digitales pueden facilitar la comprensión, la interpretación y la apropiación de la información; sin embargo, a pesar de que los formatos de los recursos educativos digitales ofrecen opciones multimediales, interactivas y de fácil acceso, por sí solos no garantizan efectividad en el logro de aprendizajes significativos ni críticos (Zapata, 2012; Quirós, 2009). Más aún, su proceso de creación de contenidos educativos originales, creativos y contextualizados, exige (tanto del profesorado como del alumnado), en primer lugar, manifestar un profundo conocimiento del tema a abordar; en segundo lugar, establecer un objetivo de enseñanza-aprendizaje, para delimitar los contenidos, y que además, permitirá escoger el recurso TIC para soportarlo, y los procedimientos metodológicos que facilitarán la aproximación de los estudiantes al objeto de estudio (Ospina, 2004 en Zapata, 2012).

En el ámbito pedagógico existen numerosas investigaciones sobre prácticas e innovaciones en contextos escolarizados relacionadas con el uso 


\section{Pérez-Ortega-Recursos Educativos Digitales}

de herramientas TIC (Area, 2008 y 2010; Castillo, 2008; Martínez y Heredia, 2010; González Mariño, 2008). Son menos las que desde la Sociología de la Educación abordan esta cuestión. Balanskat, Blamire y Kefala revisaron 17 investigaciones e informes sobre el impacto de las TIC en los sistemas escolares (en los niveles de educación primaria y secundaria) de Europa y concluyeron que, "a pesar del incremento de la disponibilidad de recursos tecnológicos en las escuelas, (...) la práctica pedagógica de los docentes en el aula no supone necesariamente una alteración sustantiva del modelo de enseñanza tradicional" (en Area, 2010, p. 6). Es decir, en el caso de educación básica, el uso de estas herramientas no ha constituido un cambio significativo en los procesos de enseñanza-aprendizaje. Esto es, observaron, por ejemplo, que los profesores usan las TIC para apoyar las pedagogías existentes, como pueden ser las exposiciones magistrales en la que se encubre ideas y dinámicas pedagógicas en las que el profesor es el único emisor válido para la transmisión de los conocimientos, mientras que el alumnado es un mero receptor.

De sus planteamientos, se concluye que el uso de las TIC en contextos educativos europeos y también de América Latina tiende a reproducir modelos de enseñanza probados $\mathrm{y}$ estos medios o instrumentos no necesariamente impulsan innovaciones pedagógicas. "El ordenador que puede contribuir a que el alumno amplíe la información, realice ejercicio o establezca alguna relación interactiva, pero con el mismo objetivo: aprender determinados contenidos y dar cuenta de ello en la evaluación correspondiente" (Marchesi y Martí, 2003, p. 115 en Area, 2008, p. 7). En otras palabras, se señala que la integración de las TIC en el currículo escolar precisa de un modelo pedagógico que otorgue sentido al uso de las mismas con perspectivas innovadoras y vinculadas a los propósitos de las competencias mediáticas (Pérez-Ortega, 2016). De esta manera, un sistema educativo rígido y poco dinámico, que da gran importancia a la memorización de los conocimientos, no propicia proyectos que busquen cambios y/o mejoras. De acuerdo con Area (2008) para lograrlo, será preciso que el currículo escolar promueva, además del acceso y uso de software y hardware, la adquisición de competencias relacionadas con búsqueda, análisis, selección y comunicación de datos e información para que el alumno la transforme en conocimiento. Es decir, en proyectos educativos que privilegien el acatamiento y el autoritarismo, y en los que los 
conocimientos se adquieran mediante ejercicios de memoria y repetición, no se promoverá la participación del alumnado, tan importante en los procesos de innovación con herramientas TIC.

Las formas de socialización han cambiado radicalmente, han adquirido una dimensión mediatizada caracterizada por la digitalización de las relaciones sociales. "Lo cotidiano se construye en esta red tecnológica y mediática, en donde los individuos tejen su camino en la sociedad, ahora mediatizada" (Riffo, 2015). En este contexto, los medios de comunicación masivos y las TIC son importantes soportes para las expresiones culturales. Son espacios idóneos para la creación, la recreación y la transmisión de la diversidad y la cantidad de contenidos (en general estandarizados) a nivel global. En la práctica resultan recursos idóneos para la diseminación (e incluso consolidación o desarraigo) de ciertas referencias culturales; no obstante, no se ha de olvidar que quienes generan contenidos son personas y/o instituciones con fines específicos. En el caso de las instituciones educativas, los recursos y los contenidos digitalizados podrían fortalecer modelos educativos que promuevan formas de explorar, representar y adquirir nuevos conocimientos. Por otro lado, las innovaciones educativas podrán potenciar la incorporación de nuevos contenidos (digitales o no) en el currículo escolar y la identificación de necesidades de formación tanto de comunicadores (y comunicólogos) como de educadores. Consecuentemente, el Proyecto fija la necesidad de identificar las demandas y las necesidades didácticas, espaciales y tecnológicas, mediante el acopio sistematizado de las mismas a través de un análisis documental.

\section{Diseño y Desarrollo del Proyecto}

En esta sección se describen las actividades que, en el marco del Proyecto se desarrollaron. Es decir, después de significar ciertas nociones, se hizo necesario diseñar las estrategias y las acciones que permitieron la articulación dialéctica y multidisciplinar que pretende el ejercicio. Sigue la descripción de la delimitación del contexto institucional, el proceso de establecimiento de objetivos de innovación educativa, así como las metas, la justificación y la evaluación de los mismos.

Teniendo como marco institucional un convenio de colaboración académica y de investigación entre el Área de Sociología del Departamento 


\section{Pérez-Ortega-Recursos Educativos Digitales}

de Educación de la UC y el área "Alfabetización mediática y cultura digital" del Departamento de Educación y Comunicación de la UAM- Xochimilco (desde septiembre de 2013), se discutió la conveniencia de promover un ejercicio teórico-práctico que buscara la creación de materiales educativos digitales por estudiantes que contribuyera a debatir sobre la importancia de forjar un marco socio-pedagógico y humanístico que promoviera este tipo de ejercicios. Así, se les propuso la grabación y la presentación pública de un video original (llamado "Cápsulas informativas") de duración máxima de cinco minutos en los que se expusieran de forma original y creativa, temáticas relacionadas con las TIC en contextos educativos.

Concretamente, el Proyecto nació de las necesidades de explorar las oportunidades que brindan las TIC como herramientas pedagógicas y sociocríticas; y como práctica del proceso de creación de innovaciones educativas, en el marco de la asignatura "Sociedad, Cultura y Educación" (cuyas temática y teorización giran en torno a la Sociología de la Educación) del primer año de los Grados de Magisterio en Educación Infantil y de Magisterio en Educación Primaria, ambas impartidas en la UC. ${ }^{1}$

La participación de los alumnos del grado de Comunicación Social (matriculados en el área de concentración "Alfabetización mediática y cultura digital") $)^{2}$ de la UAM- Xochimilco, complementó el ejercicio en tres puntos, que llevaron a: a) Estudiar (observar, escuchar e interpretar) los materiales elaborados por los alumnos de la UC, para calificar los contenidos audiovisuales con el propósito de organizarlos como estrategias para desarrollar el proceso de enseñanza-aprendizaje mediado por TIC, después; b) Sirviéndose del propio marco teórico sobre la producción de mensajes y técnicas de comunicación audiovisual en formato digital, reelaborar los discursos de manera que crearon lo que denominaron "Cápsulas informativas"; c) Discutir el papel de las TIC en ámbitos educativos; concretamente se centraron en la necesidad de formarse en la creación de nuevos contenidos y el uso de herramientas de tecnológicas y digitales para su difusión en contextos educativos.

De manera sintética, se establece que las metas que se propuso alcanzar el Proyecto fueron: a) Desarrollar debates teóricos sobre el uso de las TIC en contextos educativos como facilitadores del proceso de enseñanzaaprendizaje en cuanto herramientas multiplicadoras de lenguajes didácticos y por ello, capaces de motivar cambios y o mejoras educativas; b) 
Experimentar el diseño, la pre-producción, producir y post-producir) y la difusión (presentación pública) de productos audiovisuales originales como posibles innovaciones educativa; c) Evaluación grupal de los alcances del Proyecto.

El establecimiento de estas metas llevó a la delimitación y a la organización de las actividades alrededor del video, ordenadas en temporalidades diferenciadas entre sí y en contextos educativos específicos, de la forma que a continuación se describe; sin embargo antes se anota que los puntos 1 y 2 fueron desarrollados en ambas universidades en tiempos diferentes (en función de la organización de los calendarios en cada una). Luego, desde el 3 y hasta el 6 describen los pasos que realizaron los alumnos de los grados de Magisterio en Educación Infantil y Magisterio en Educación Primaria la UC; mientras a partir del punto 7 se relatan las actividades en las que participaron los del grado de Comunicación Social de la UAM - Xochimilco.

1. Presentación de los objetivos, metodología y evaluación del proyecto en ambas universidades por parte de los profesores responsables de la asignatura (UC) y del área de concentración (UAM - Xochimilco).

2. Delimitación teórica de los conceptos subyacentes, mediante análisis sistematizado y posterior debate colectivo de los mismos.

3. Preproducción. Delimitación de los contenidos y reflexión sobre la forma de abordar el mensaje a ofrecer mediante un canal audiovisual. Para ello fue preciso la identificación de los recursos humanos y materiales para la elaboración del mismo. En síntesis, esta actividad exigió la elaboración del guion (o storyboard) y la selección (o scouting) de locaciones y horarios de grabación del material.

4. Producción. Proceso de grabación del material en las locaciones y horarios elegidos.

5. Postproducción. Edición casera y con recursos digitales (programas) de libre acceso. Concretamente, para la edición del material se usaron los siguientes programas de edición de video gratuitos para Windows: Windows Movie Maker, VirtualDub, Wax, Avidemux, FFMpeg, Blender, entre otros. Destaca que hasta el momento, las acciones descritas fueron desarrolladas por alumnos de Magisterio en Educación Infantil y Magisterio en Educación Primaria de la Universidad de Cantabria, por lo que no cuentan con competencias de manejo de TIC formalizadas. 


\section{Pérez-Ortega-Recursos Educativos Digitales}

6. Difusión. Presentación pública del video en la UC y evaluación del producto. Cabe señalar que en esta parte del proyecto se revisó y calificó el trabajo colectivo de los alumnos como el resultado de un proceso de elaboración de un discurso audiovisual que pretendió dotar información (a otros universitarios) sobre varios conceptos vinculados a las TIC de forma original y creativa. Por ello, se prestó más atención al contenido que al formato.

7. Visualización de los productos finales elaborados por los alumnos de la UC con la finalidad de reflexionar en manera de mejorar el formato, de manera que el objetivo fue: "Reelaborar el formato de presentación de los productos audiovisuales elaborados por alumnos de la UC sobre conceptos relacionados con TIC de manera que se favorezca visual y auditivamente la comprensión, la apropiación y la significación de la información en contextos transnacionales. Siendo el reto último la elaboración de cápsulas informativas"

8. Con el objetivo de incrementar la calidad audiovisual de los materiales originales, los alumnos de la UAM -Xochimilco se centraron en la resolución de problemáticas del lenguaje gráfico y de audio. Para ello, se revisó y se calificó el material en función de su utilidad como mensaje multimedia que recoge y reproduce un determinado contexto enunciativo; es decir, al analizar los videos realizados por los alumnos de la UC se revelaron, además de los recursos técnicos que usaron, sus puntos de vista, intereses, gustos o necesidades como realizadores.

9. Luego, se editó y se montó en un nuevo soporte, en forma de "Cápsulas informativas" apto para su reproducción de Windows; para ello utilizaron programas licenciados como el software de Adobe ${ }^{\circledR}$ Photoshop ${ }^{\circledR}$ con la versión CS5. También se usaron otras herramientas de diseño gráfico

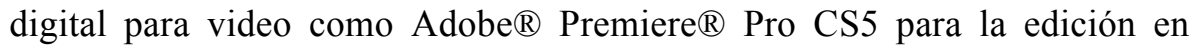
diversas plataformas y AVID Technology disponibles en la UAM Xochimilco. Se destaca que también se permitió el uso de las herramientas Inkscape, GIMP y Jahshaka por ser software opensource y con elevada calidad profesional.

10. La presentación pública de las "Cápsulas informativas" estuvo acompañada de un debate teórico que se centró en dos cuestiones: a) Señalar que su utilidad como materiales educativos radica en su capacidad de re-producir ciertos aspectos de la realidad en que fueron elaborados. Es 
decir, permiten observar, estudiar y analizar situaciones y contextos a través de imágenes y sonidos como representaciones colectivas. b) Reconocer que el uso de videos en el proceso de enseñanza-aprendizaje es una tendencia que se va afianzando, por lo que es preciso responder a la necesidad de formación en cuanto a competencias de alfabetización audiovisual entendida como la capacidad para leer, construir y comprender signos, símbolos y mensajes visuales a partir del reconocimiento de su estructura formal (Dondis, 1990).

Finalmente, se realizó la evaluación de las "Capsulas informativas" como producto final audiovisual. En este punto se subraya que la calificación de los videos no constituyó la valoración de los objetivos y resultados del Proyecto. La primera se realizó como lo establecen sus programaciones docentes, mientras que la segunda precisó realizar debates grupales y una encuesta para los alumnos de las dos instituciones.

En la siguiente sección se expone la discusión de los logros, limitaciones y resultados del Proyecto siguiendo la metodología socio-crítica. Es decir, para identificar sus metas se contrastaron e interpretaron los resultados de los cuestionarios con las respuestas espontáneas surgidas de dos debates dirigidos (uno en la UC -43 participantes- y otro en la UAM- Xochimilco 26 participantes-) y que precisaron el uso de un guion previamente elaborado. ${ }^{3}$

\section{Interpretación de los Resultados Del Proyecto}

Como se ha manifestado, el texto considera que proceso de enseñanzaaprendizaje es un conjunto de manifestaciones culturales que tienden a la reproducción de las estructuras y de los valores sociales dominantes. Por ello, específicamente en aquel que promueva la formación en habilidades y destrezas de acceso y uso de TIC, es necesario observar que las programaciones docentes lo incluyan; ya que el desarrollo de prácticas mediadas por los recursos que estas tecnologías podrían (mediante trabajo continuado entre profesores y alumnos) lograr cambios y/o mejoras en las experiencias de enseñanza-aprendizaje. Esta sección se centra la interpretación de cinco aspectos que se considera permiten identificar la manera en que este aporta a la discusión sobre las posibilidades de los recursos educativos digitales para cambiar y/o mejorar algunas didácticas: 


\section{Pérez-Ortega-Recursos Educativos Digitales}

1. Desarrollo de contenidos educativos originales en formato digital.

2. Dotación de técnicas para elaborar recursos educativos digitales.

3. TIC como recursos didácticos.

4. Uso de TIC para promover cambios y/o mejoras educativas.

5. Uso de recursos TIC en futuras experiencias profesionales docentes.

En opinión de los estudiantes, el Proyecto permite reflexionar sobre la posibilidad de desarrollar nuevos y originales contenidos educativos con herramientas TIC. Manifiestan que, a pesar de la presencia de numerosas argumentaciones que reconocen la importancia de crear nuevas prácticas y metodologías con TIC en contextos escolarizados, la estructura del modelo de educación superior no facilita, en la práctica (y de forma generalizada) el uso significativo ni innovador de estas. Por ello, estas experiencias son útiles porque potencien reflexiones y permiten experimentar el proceso de elaboración de materiales educativos digitalizados.

En concreto, las percepciones de los alumnos permitieron reflexionar sobre las causas y los ritmos de los cambios planificados y dirigidos en las dinámicas escolares que no necesariamente responden a las reformas educativas, sino que resultan de una combinación de transformaciones culturales, económicas, sociológicas y generacionales que tienen lugar en los centros educativos y reflejan una lógica interna (López- Yáñez, 2010). El Gráfico 1 recoge las opiniones de los estudiantes que participaron en el proyecto sobre la meta primera del mismo. 


\section{¿PERMITE REFLEXIONAR SOBRE LA POSIBILIDAD DE DESARROLLAR NUEVOS Y ORIGINALES CONTENIDOS EDUCATIVOS CON HERRAMIENTAS TIC?}

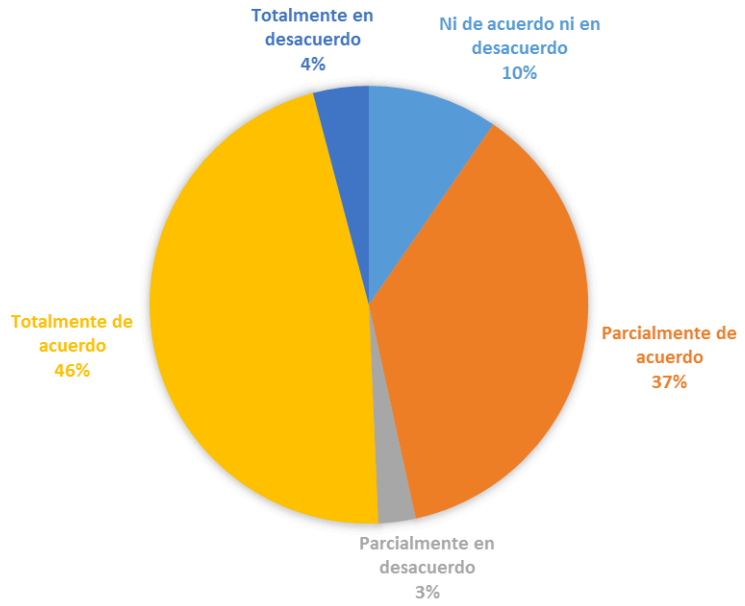

Figura 1. Desarrollo de contenidos educativos originales en formato digital Fuente: Elaboración propia

Después, sobre la promoción de habilidades de los alumnos para la elaboración de contenidos digitales, los alumnos subrayaron las dificultades técnicas. Los de la UC manifestaron su escasa formación sobre el uso de los recursos software de libre acceso. Sobre las complicaciones organizativas y de gestión, todos los alumnos señalaron la gran cantidad de tiempo que exigió elaborar el producto. Finalmente, en cuanto a las dificultades didácticas, manifestaron conflictos en el uso de un lenguaje común entre dos contextos culturalmente diferentes entre sí. El Gráfico 2 refleja la opinión de los alumnos sobre la potencialidad del proyecto para dotarlos de destrezas y/o habilidades para desarrollar recursos educativos digitales. 


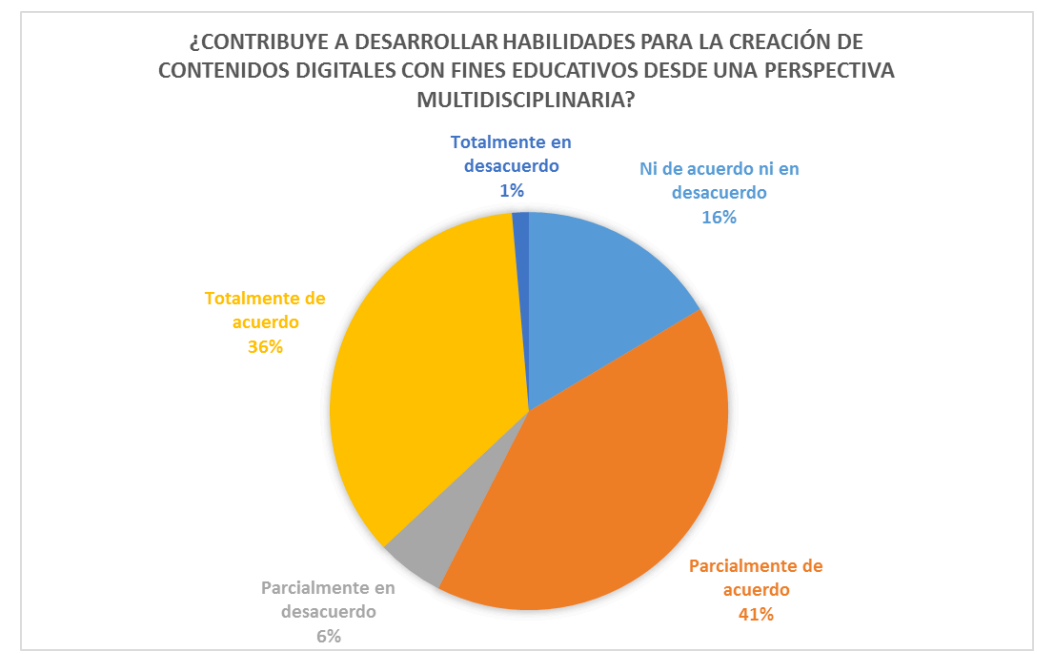

Figura 2. Dotación de técnicas para elaborar recursos educativos digitales Fuente: elaboración propia

Respecto al uso de TIC en contextos educativos, los alumnos consideran que el proyecto fue una oportunidad para vincular sus conocimientos tecnológicos previos (unos adquiridos mediante el uso cotidiano de gadgets, por ejemplo y otros como parte de su formación universitaria) con temáticas puntuales del programa docente de los grados que cursan. El Gráfico 3 recoge la opinión de los participantes sobre el uso de videos como medios para difundir contenidos educativos nuevos y originales. 


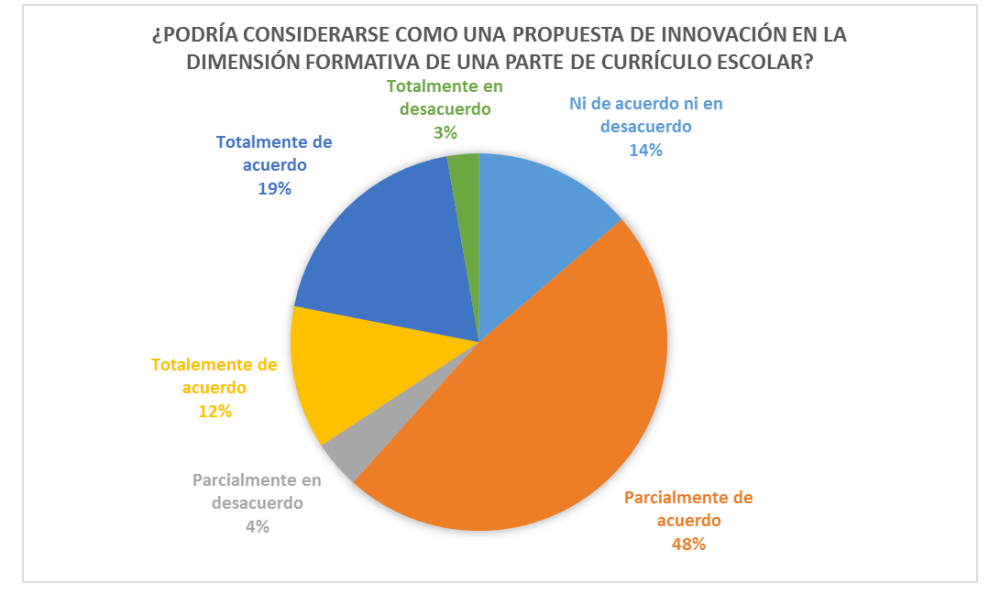

Figura 3. TIC como recursos didácticos

Fuente: elaboración propia

Sobre el potencial de las TIC para impulsar cambios y/o mejoras en las metodologías docentes, los alumnos consideran que el Proyecto promueve la reflexión sobre las innovaciones en contextos educativos; sin embargo, manifiestan escepticismo sobre la manera en que la elaboración de sus propias grabaciones audiovisuales podría incidir en la estructura del currículo escolar. Es de resaltar que los alumnos también reconocen que la actividad podría significarse como ensayo de cambio y/o mejora educativa a largo plazo.

Siguiendo a López-Yáñez (2010), sobre este punto se reflexiona sobre los mecanismos y las dinámicas sociales que subyacen en las prácticas de innovación educativa. Es decir, es reconoce la importancia de observar las tramas sociales e ideológicas detrás de los procesos de innovación y que serán las que faciliten su perduración en tiempos prologados. Para este autor la sostenibilidad de proyectos educativos innovadores se vincula directamente con la presencia de condiciones organizativas (sobre todo las informales) caracterizadas por la apropiación y valoración positiva de significados que subyacen en los cambios y/o mejoras, por parte de quienes los llevan a cabo.

El Gráfico 4 relata la opinión de los alumnos de la UC y de la UAMXochimilco sobre la cuantía de esta actividad como propulsora de 


\section{Pérez-Ortega-Recursos Educativos Digitales}

innovaciones educativas $\mathrm{y}$, luego, de su futura incorporación a las programaciones docentes.

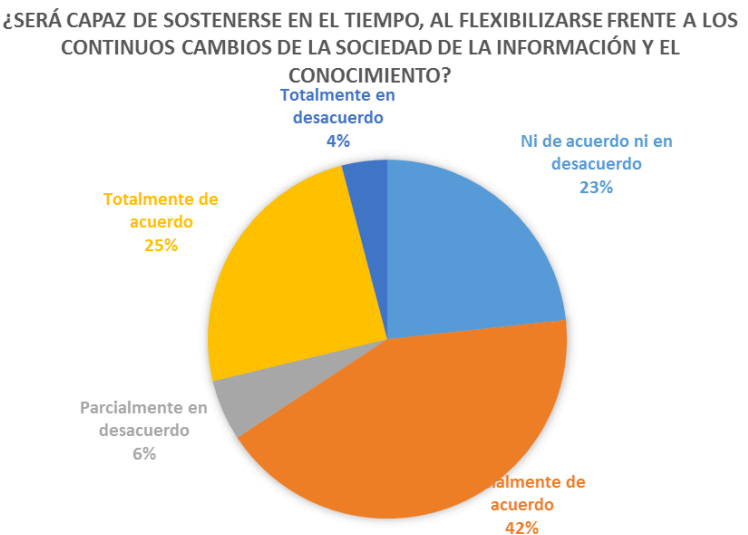

Figura 4. Uso de TIC para impulsar cambios y/o mejoras educativas Fuente: elaboración propia

Finalmente, sobre la reproducción de este tipo de actividades en futuros contextos laborales, los alumnos manifestaron su disposición a probar replicarlos, siempre que se cuente con recursos tecnológicos suficientes. Los alumnos de la UAM- Xochimilco señalaron que para lograr un intercambio significativo entre los protagonistas del proceso de enseñanza-aprendizaje, será fundamental considerar que el lenguaje audiovisual (independientemente del contenido). Es decir, se han de considerar dos cuestiones fundamentales: a) las particularidades de los códigos comunicativos multimedia y; b) los posibles contextos de enunciación. De esto se deduce que los recursos educativos digitales han de ser flexibles en cuanto que permitirán la modificación de su contenido de acuerdo a las necesidades específicas de los diferentes contextos educativos. El Gráfico 5 expone la opinión de los alumnos sobre la posibilidad de usar documentos digitales con fines educativos de elaboración propia como recursos complementarios y facilitadores del proceso de enseñanza aprendizaje. 


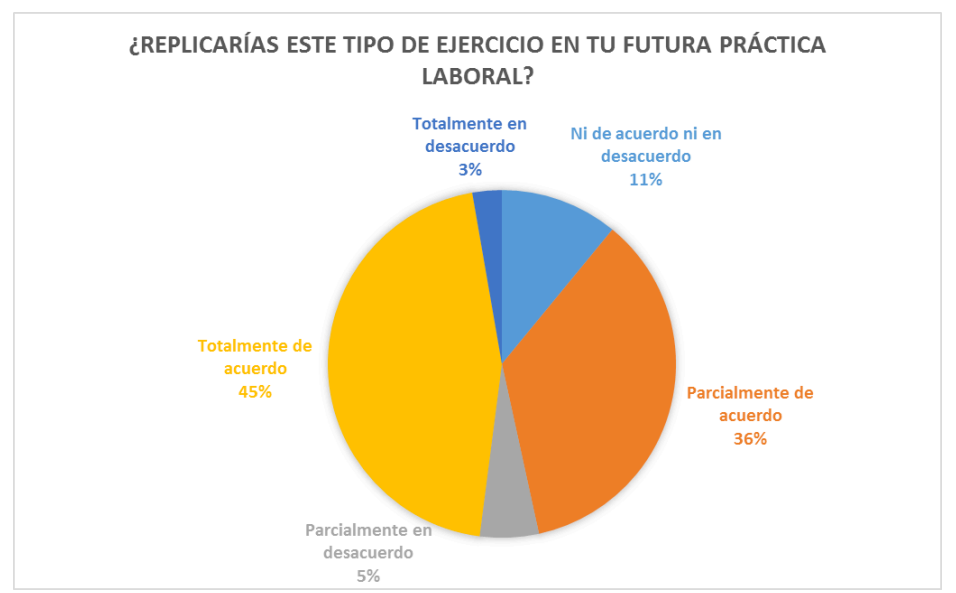

Figura 5. Uso de recursos TIC en futuras experiencias profesionales docentes Fuente: elaboración propia

Una contribución de este Proyecto es que, en opinión de los alumnos, su uso podría favorecer cambios y/o mejoras en las metodologías educativas y en la formación de futuros profesores. Sin embargo, es importante destacar, como lo hace González Mariño (2008, p. 7):

No es sólo la inclusión de TIC en la enseñanza lo que le da el carácter innovador, la innovación educativa debe verse desde una perspectiva mucho más amplia e integral, donde la combinación de los medios tecnológicos adecuados y un diseño didáctico basado en las necesidades específicas de aprendizaje de acuerdo al contexto.

En el contexto de la sociedad de la información y el conocimiento, la formación de profesores ha de responder a las nuevas configuraciones de la tecnologización de las interacciones sociales. En cuanto la alfabetización digital, se considera que será muy importante reflexionar sobre las repercusiones de estas transformaciones tanto en el campo de la investigación como en la práctica curricular. Es decir, se entiende como lo hace Barrón e Ysunza (2003) que la discusión de la finalidad educativa de promover la creación de materiales didácticos digitales ha de incluir el debate sobre la conceptualización y las estrategias para la formulación del 


\section{Pérez-Ortega-Recursos Educativos Digitales}

perfil profesional, lo que llevará a identifica un campo de problemas que tocan la pertinencia actual de las estructuras curriculares.

\section{Reflexiones Finales}

Los actuales entornos socio-educativos exigen potenciar el desarrollo de habilidades avanzadas de pensamiento crítico para poder comprender y usar la información transmitida por las TIC. En contextos de educación superior, las programaciones docentes se están transformando para intentar dar cabida a enfoques que promuevan el uso de herramientas tecnológicas; sin embargo, estas propuestas por sí solas, no representan cambios y/o mejoras en las prácticas docentes. En Europa, diferentes estudios sobre innovación educativa han demostrado que la promoción de cambios y/o mejoras educativas con TIC en los currículos escolares no conlleva su ejecución extendida e igualitaria. Para Tondeur, Van Braak y Valcke (2007, p.28) no necesariamente hay coherencia entre las sugerencias del texto curricular y las dinámicas escolares, porque en la práctica, el uso de TIC implica la conjunción de otros factores, como por ejemplo la formación de los recursos humanos y la presencia de recursos tecnológicos, pero sobre todo de la participación activa tanto de profesores como de alumnos. En otras palabras, el uso de TIC en contextos educativos puede servir para innovar en aspectos del proceso de enseñanza-aprendizaje; sin embargo la observación del Proyecto manifestó:

En primer lugar, la propuesta de elaborar recursos digitales como materiales didácticos no es una actividad nueva. Sin embargo, el Proyecto señaló que los cambios y/o mejoras con TIC en las dinámicas escolares resultarán luego de conocer y apropiar de las potencialidades de estos recursos como medios para transmitir determinados contenidos. Por ello han de resignificarse como herramientas útiles para la expresión de otros lenguajes, otras perspectivas académicas y otras culturas; así como para la promoción de relaciones igualitarias entre alumnos y profesores. Es decir, los actuales contextos educativos digitalizados exigen la formación de usuarios capaces de comprender un determinado código simbólico (o lenguaje significativo) para interactuar en ellos (Riffo, 2015). En palabras de Tello y Aguaded (2009, p. 41), "la incorporación de las TIC supone mucho más que dotar a los centros de equipamiento e infraestructuras: además, es 
necesario favorecer y desarrollar la capacidad de reflexionar sobre la información recibida". Por si fuera poco, la introducción de las TIC en las dinámicas escolares (ya no en el currículo escolar) exige contar con políticas que impulsen la alfabetización de las tecnologías digitales. Más allá del manejo del hardware y del software, será importante puntualizar las reflexiones académicas en torno a los códigos de los lenguajes digitalizados y las formas comunicativas mediados por TIC en contextos educativos.

En segundo lugar, la inclusión de prácticas con TIC en formaciones de grado es insuficiente para cambiar y/o mejorar metodologías docentes caracterizadas por la reproducción de esquemas en los que el proceso de enseñanza- aprendizaje privilegia el rol del profesor como principal transmisor de la información. Específicamente, actividades como las proyecto podrían significar alguna innovación en medida en que se discutan las limitaciones y las potencialidades de las TIC como canales para las manifestaciones culturales que se producen y reproducen en entornos escolarizados. Siguiendo a Tello y Aguaded (2009, p. 45) se afirma que la labor del profesor consiste en "hacer que el alumnado se permita el mismo la posibilidad de buscar su propio bagaje cognitivo y relacional, en un mundo plural".

Partiendo del reconocimiento de las diferencia socio-culturales, políticos y económicos que caracterizan los contextos escolares observados se señala que la integración de TIC como herramientas simbólicas con capacidad de producir discursos alternativos al dominante, trae consigo exigencias relacionadas con la transformación de las representaciones socio-culturales de quienes dan vida a las instituciones y desarrollan los modelos de educación superior, de manera que a futuro en el currículo escolar de educación superior sea posible introducir debates teóricos y ejercicios prácticos que promuevan la observación de las TIC como fenómenos culturales, además contemplar contenidos que apunten al manejo tanto del software como del hardware.

Finalmente, se señala las actividades que contempló el Proyecto no encuentran expresión en los documentos curriculares oficiales. En las programaciones docentes de educación superior si bien se resalta la importancia de formar en el uso de recursos TIC, es poco frecuente encontrar manifestaciones claras sobre la importancia de promover el análisis y la interpretación de la información digitalizada para transformarla 


\section{Pérez-Ortega-Recursos Educativos Digitales}

en conocimientos que permitirán la interpretación de contextos análogos o mediados por TIC. Su incorporación exigiría, entre otros, implantar acciones que lleven a la investigación teórica o la observación sistematizada de proyectos que promuevan debates académicos sobre la alfabetización digital, la cultura mediática, los modelos didácticos con TIC, el trabajo colaborativo y multidisciplinario entre diferentes instituciones. En otras palabras, la modificación de la estructura de los programas docentes sobre el uso de TIC en contextos escolarizados precisa llevar a cabo pequeños cambios en las dinámicas de los centros educativos.

\section{Notas}

${ }^{1}$ Sociedad, Cultura y Educación es una asignatura perteneciente al área de Sociología del Departamento de Educación de la Universidad de Cantabria. Se imparte bajo la modalidad de presencial en el primer cuatrimestre de los grados de Magisterio en Educación Infantil y Magisterio en Educación Primaria. Constituye 6 créditos académicos ECTS. Más información en: web.unican.es.

${ }^{2}$ El área de Alfabetización Mediática y Cultura Digital se define como el espacio de aprendizaje de las herramientas y metodologías para que los alumnos identifiquen las problemáticas de la Sociedad de la Información. Más información en: http://dcsh.xoc.uam.mx/liccom/.

${ }^{3}$ Respecto a los cuestionarios y los debates se señalan algunas precisiones: El cuestionario incluyó diez preguntas cerradas, que recogió datos como edad, sexo, carrera y lugar de residencia (España y México), pero en el texto se manifiestan y discuten los resultados que se relacionan directamente con la experiencia de creación de recursos digitales como materiales didácticos. Luego, se usaron todos los cuestionarios porque éstos fueron cumplimentados por la totalidad de los alumnos que participaron del proyecto, probablemente debido a que se realizaron en presencia del docente-investigador y la actividad formaba parte de la evaluación de una asignatura obligatoria.

Sobre los debates grupales, se destaca se realizaron únicamente con los alumnos que participaron en el proyecto en su segunda edición y con el objetivo de contrastar la información recogida a través de los cuestionarios.

\section{Referencias}

Area, M. (2008). La innovación pedagógica con TIC y el desarrollo de las competencias informacionales y digitales. Investigación en la escuela, 64, 6-17. 
Barrón, C. \& Ysunza, M. (2003). Currículum y formación profesional. En A. Barriga (Coord.), La investigación curricular en México en la década de los noventa). Ciudad de México: Consejo Mexicano de Investigación Educativa, AC. 175 - 179

Blanco, N. (1994). Las intenciones educativas. En J. F. Ángulo \& N. Blanco (Coord.). Teoría y desarrollo del currículum Málaga: Ediciones Aljibe Crítica y Fundamentos. 210 - 240.

Dondis, D. A. (1990). La sintaxis de la imagen. Barcelona: Gustavo Gili. Durall, E., Gros, B., Maina, M., Johnson, L. \& Adams, S. (2012).

Perspectivas tecnológicas: educación superior en Iberoamérica 2012-2017. Austin, Texas: The New Media Consortium.

González Mariño, J. C. (2008). TIC y la transformación de la práctica educativa en el contexto de las sociedades del conocimiento. Revista de Universidad y Sociedad del Conocimiento (RUSC), 5(2). UOC. Recuperado de http://www.uoc.edu/rusc/5/2/dt/esp/ gonzalez.pdff Guerrero, I. \& Kalman, J. (2010). La inserción de la tecnología en el aula: Estabilidad y procesos instituyentes en la práctica docente. Revista Brasileira de Investigación Educativa, 15, 44, 213-299.

Gutiérrez, A. \& Tyner, K. (2012). Presentación. Alfabetización mediática en contextos múltiples. Media Literacy in Multiple Contexts. Revista Comunicar, 38, XIX, 10-12. doi: 10.3916/C38-2012-02-00

López - Yañez, J. (2010). Sostenibilidad de las innovaciones en los centros escolares: sus bases institucionales. Profesorado. Revista de currículum y formación del profesorado, 14, 1, 9-28.

Martínez González, Raquel-Amaya (2007). La investigación en la práctica educativa: Guía metodológica de investigación para el diagnóstico y evaluación en los centros docentes. Madrid: Ministerio de Educación y Ciencia. Recuperado de https://sede.educacion.gob.es/publiventa/PdfServlet?pdf=VP12309.p df\&area $=\mathrm{E}$

Perazzo, M. I. (2008). La ruta de la alfabetización digital en la educación superior. Revista Universidad y Sociedad del Conocimiento (RUSC), 5(1). Recuperado de http://rusc.uoc.edurusc.

Pérez, M. A. \& Delgado, A. (2012). De la competencia digital y audiovisual a la competencia mediática: dimensiones e indicadores. Revista Comunicar. Revista Científica de Educomunicación, 39, 25-34. 
Pérez-Ortega, I. (2016). La competencia mediática en el currículo escolar: ¿espacio para innovaciones educativas con tecnologías de la información y la comunicación? Revista Innovación educativa, 16(70). 61-83.

Quirós, E. (2009). Recursos didácticos digitales: medios innovadores para el trabajo cooperativo en línea. Revista Electrónic@Educare. XIII(2), 47-62.

Riffo, I. (2015). La cultura mediática. Reflexiones y perspectivas - España. RevistaComunic@cción,6(2). Recuperado de http://www.comunicacionunap.com/index.php/rev/article/view/70/73

Romero, J. \& Luis, A. (2008). El conocimiento socio-geográfico en la escuela: Las tensiones inherentes a la transmisión de cultura institucionalizada y los dilemas de la educación para la democracia en este mundo globalizado. Scripta Nova. Revista electrónica de geografía y ciencias sociales, XII(270), 123- 125.

Tello, J. \& Aguaded, J. I. (2009). Desarrollo profesional docente ante los nuevos retos de las tecnologías de la información y la comunicación en los centros educativos. Pixel Bit. Revista de Medios y Educación, 34, 31- 47.

Tondeur J., Van Braak, J. \& Valcke, M. (2007). Curricula and the use of ITC: two words apart? British Journal of Educational Technology, 38. doi: 10.1111/j.1467-8535.2006.00680.x

Zapara, M. (2012). Recursos educativos digitales: conceptos básicos. Programa Integración de Tecnologías a la docencia. Universidad de Antioquia. Recuperado de http://aprendeenlinea.udea.edu.co/boa/contenidos.php/.../845/estilo/... $=/ 1 /$ contenido

Isabel Pérez-Ortega is professor at University of Cantabria, Spain Contact Address: portegami@unican.es 